\title{
Rotating and Counterrotating Relativistic thin Disks as Sources of Stationary Electrovacuum Spacetimes
}

\author{
Gonzalo García-Reyes \\ Universidad Tecnológica de Pereira, Departamento de Física, A. A. 97, Pereira, Colombia \\ and Guillermo A. González \\ Escuela de Física, Universidad Industrial de Santander A.A. 678, Bucaramanga, Colombia
}

Received on 7 December, 2006

\begin{abstract}
A detailed study is presented of the counterrotating model (CRM) for electrovacuum stationary axially symmetric relativistic thin disks of infinite extension without radial stress, in the case when the eigenvalues of the energy-momentum tensor of the disk are real quantities, so that there is not heat flow. We find a general constraint over the counterrotating tangential velocities needed to cast the surface energy-momentum tensor of the disk as the superposition of two counterrotating charged dust fluids. We then show that, in some cases, this constraint can be satisfied if we take the two counterrotating tangential velocities as equal and opposite or by taking the two counterrotating streams as circulating along electro-geodesics. However, we show that, in general, it is not possible to take the two counterrotating fluids as circulating along electro-geodesics nor take the two counterrotating tangential velocities as equal and opposite. A simple family of models of counterrotating charged disks based on the Kerr-Newman solution are considered where we obtain some disks with a CRM well behaved. We also show that the disks constructed from the Kerr-Newman solution can be interpreted, for all the values of parameters, as a matter distribution with currents and purely azimuthal pressure without heat flow. The models are constructed using the well-known "displace, cut and reflect" method extended to solutions of vacuum Einstein-Maxwell equations. We obtain, in all the cases, counterrotating Kerr-Newman disks that are in agreement with all the energy conditions.
\end{abstract}

Keywords: General relativity; Thin disks; Exact solutions; Einstein-Maxwell equations

\section{INTRODUCTION}

Several methods are known to exactly solve the Einstein and Einstein-Maxwell field equations, or to generate new exact solutions from simple known solutions [1]. However, the above mentioned methods in general lead to solutions without a clear physical interpretation or to solutions that depend on many parameters without a clear physical meaning. Accordingly, it is of importance to have some appropriate procedures to obtain physical interpretations of these exact solutions. So, in the past years such procedures have been developed for static and stationary axially symmetric solutions in terms of thin and, more recently, thick disk models.

Stationary or static axially symmetric exact solutions of Einstein equations describing relativistic thin disks are of great astrophysical importance since they can be used as models of certain stars, galaxies and accretion disks. These were first studied by Bonnor and Sackfield [2], obtaining pressureless static disks, and then by Morgan and Morgan, obtaining static disks with and without radial pressure [3, 4]. In connection with gravitational collapse, disks were first studied by Chamorro, Gregory, and Stewart. Also thin disks with radial tension were considered [6]. Several classes of exact solutions of the Einstein field equations corresponding to static thin disks with or without radial pressure have been obtained by different authors [7-15].

Rotating thin disks that can be considered as a source of a Kerr metric were presented by Bičák and Ledvinka [16], while rotating disks with heat flow were studied by González and Letelier [17]. The nonlinear superposition of a disk and a black hole was first obtained by Lemos and Letelier [10]. Perfect fluid disks with halos were studied by Vogt and Letelier [18]. The stability of some general relativistic thin disks models using a first order perturbation of the energy-momentum tensor was investigated by Ujevic and Letelier [19].

González and Letelier [20] constructed models of static relativistic thick disks in various coordinate systems. Although the disks have constant thickness, the matter density decreases rapidly with radius and the $z$ coordinate, and in principle they also can be used to represent both the disk part and the central bulges of galaxies. Also Vogt and Letelier [21] considered more realistic three-dimensional models for the gravitational field of Galaxies in the General Relativistic context. Essentially they formulate the General Relativistic versions in isotropic coordinates of the potential-density pairs deduced by Miyamoto and Nagai [22, 23] and Satoh [24].

Disk sources for stationary axially symmetric spacetimes with magnetic fields are also of astrophysical importance mainly in the study of neutron stars, white dwarfs and galaxy formation. Although disks with electric fields do not have clear astrophysical importance, their study may be of interest in the context of exact solutions. Thin disks have been discussed as sources for Kerr-Newman fields [25], magnetostatic axisymmetric fields [26], conformastationary metrics [27], while models of electrovacuum static counterrotating dust disks were presented in [28]. Charged perfect fluid disks were also studied by Vogt and Letelier [29], and charged perfect fluid disks as sources of static and Taub-NUT-type spacetimes by García-Reyes and González [30, 31].

In all the above cases, the disks are obtained by an "inverse problem" approach, called by Synge the "g-method" 
[32]. The method works as follows: a solution of the vacuum Einstein equations is taken, such that there is a discontinuity in the derivatives of the metric tensor on the plane of the disk, and the energy-momentum tensor is obtained from the Einstein equations. The physical properties of the matter distribution are then studied by an analysis of the surface energy-momentum tensor so obtained. Another approach to generate disks is by solving the Einstein equations given a source (energy-momentum tensor). Essentially, they are obtained by solving a Riemann-Hilbert problem and are highly nontrivial [33-39]. A review of this kind of disks solutions to the Einstein-Maxwell equations was presented by Klein in [40].

Now, when the inverse problem approach is used for static electrovacuum spacetimes, the energy-momentum tensor is diagonal and its analysis is direct and, except for the dust disks, all the obtained disks have anisotropic sources with azimuthal stress different from the radial stress. On the other hand, when the considered spacetime is stationary, the obtained energy-momentum tensor is non-diagonal and the analysis of its physical content is more involved and, in general, the obtained source is not only anisotropic but with nonzero heat flow. Due to this fact, there are very few works about of stationary electrovacuum disks and they are limited to disks obtained with solutions that lead to disks without heat flow $[25,31]$.

The necessary condition to obtain a thin disk without heat flow is that the eigenvalues of the energy-momentum tensor must be real quantities, which can be only for very few known electrovacuum solutions. In [31] we consider a Taub-NUT type solution such that the energy-momentum tensor can be written as an upper right triangular matrix, so that the diagonalization is trivial and the eigenvalues are real quantities. However, the obtained disks are not really rotating disks since the spatial components of their velocity vectors are zero with respect to the coordinates and so the disks are "locally statics". The first true rotating electrovacuum thin disks were obtained by Ledvinka, Bičák, and Žofka [25] by applying the "displace, cut and reflect" method to the Kerr-Newman solution. The so obtained disks have no radial pressure and no heat flow. However, the authors do not show if the eigenvalues of the momentum-energy tensor are real quantities for all the values of the parameters, is that, if these disks can always be interpreted as a matter distribution with currents and purely azimuthal pressure, or if there are some case where can exist nonzero heat flow (complex eigenvalues).

The above disks can also be interpreted as made of two counterrotating streams of moving charged particles, as was also indicated in [25]. Now, in order to do this interpretation, the counterrotating tangential velocities of the two streams must to satisfy a constraint, which in general is not satisfied for disks obtained from generic stationary electrovacuum solutions. In [25] the authors take the two counterrotating streams as circulating along electro-geodesics, but they do not show if such decomposition can be done. In addition, as we will show in this paper, in general the electro-geodesics motion do not agree with the above mentioned constraint and so it is necessary to consider another possibility for the com- plete determination of the counterrotating tangential velocities. Another possibility, also commonly assumed, is to take the two counterrotating velocities as equal and opposites but, as we will show, in general the counterrotating velocities are not completely determined by the constraint, so that the corresponding interpretation as two counterrotating streams is not possible.

The above interpretation is obtained by means of the Counterrotating Model (CRM) in which the energy-momentum tensor of the source is expressed as the superposition of two counterrotating perfect fluids. Now, even though this interpretation can be seen as merely theoretical, there are observational evidence of disks made of streams of rotating and counterrotating matter (see, for instance, [41-43]). These disks are made of stars and gas so that they are disks with pressure. Nevertheless, as is suggested in [42], the preexisting galaxies have a component originally constituted mainly by a gas free stellar disk, i.e., collisionless matter or dust. A detailed study of the CRM for generic relativistic static thin disks was presented in [15] for the vacuum case, whereas the extension for static electrovacuum disks was presented in [28, 30]. On the other hand, the CRM for stationary thin disks has not been completely developed, neither for the vacuum case, and only a preliminary version of it was presented in [17] for the case of stationary thin disks without heat flow and with positive radial stress (pressure).

The purpose of the present paper is twofold. In first instance, we present a detailed analysis of the energymomentum tensor and the surface current density for electrovacuum stationary axially symmetric relativistic thin disks of infinite extension without radial stress, in the case when the energy-momentum tensor of the disks can be diagonalized, so that there is not heat flow. And, in the second place, we present the complete study of the Counterrotating Model for these stationary thin disks. The paper is structured as follows. In Sec. II we present a summary of the procedure to obtain models of rotating thin disks with a purely azimuthal pressure and currents, using the well-known "displace, cut and reflect" method extended to solutions of Einstein-Maxwell equations, in the case when the eigenvalues of the energy-momentum tensor of the disk are real quantities. In particular, we obtain expressions for the surface energy-momentum tensor and the surface current density of the disks.

In Sec. III the disks are interpreted in terms of the counterrotating model (CRM). We find the general constraint over the counterrotating tangential velocities needed to cast the surface energy-momentum tensor of the disk as the superposition of two counterrotating charged dust fluids. We then show that this constraint can be satisfied if we take the two counterrotating tangential velocities as equal and opposite as well as by taking the two counterrotating streams as circulating along electro-geodesics. However, we show that, in general, it is not possible to take the two counterrotating fluids as circulating along electro-geodesics nor take the two counterrotating tangential velocities as equal and opposite. We also find explicit expressions for the energy densities, current densities and velocities of the two counterrotating fluids.

In the following section, Sec. IV, we consider a family 
of models of counterrotating charged dust disks based on the Kerr-Newman metric, perhaps the only simple electrovacuum solution that lead to stationary thin disks without heat flow. We show that for Kerr-Newman fields the eigenvalues of the energy-momentum tensor of the disks are always real quantities, for all the values of the parameters, and so they do not present heat flow in any case. We also analyze the CRM for these disks and study the tangential velocities, energy and electric charge densities of both streams when the two fluids move along electrogeodesics and when they move with equal and opposite velocities. Also the stability against radial perturbation is analyzed in both of the cases. Finally, in Sec. V, we summarize our main results.

\section{ELECTROVACUUM ROTATING RELATIVISTIC THIN DISKS}

A sufficiently general metric for our purposes can be written as the Weyl-Lewis-Papapetrou line element [1],

$d s^{2}=-e^{2 \Psi}(d t+\mathcal{W} d \varphi)^{2}+e^{-2 \Psi}\left[r^{2} d \varphi^{2}+e^{2 \Lambda}\left(d r^{2}+d z^{2}\right)\right]$, where we use for the coordinates the notation $\left(x^{0}, x^{1}, x^{2}, x^{3}\right)=$ $(t, \varphi, r, z)$, and $\Psi, \mathcal{W}$, and $\Lambda$ are functions of $r$ and $z$ only. The vacuum Einstein-Maxwell equations, in geometric units in which $8 \pi G=c=\mu_{0}=\varepsilon_{0}=1$, are given by

$$
\begin{aligned}
& G_{a b}=T_{a b}, \\
& F_{; b}^{a b}=0,
\end{aligned}
$$

with

$$
\begin{aligned}
T_{a b} & =F_{a c} F_{b}{ }^{c}-\frac{1}{4} g_{a b} F_{c d} F^{c d}, \\
F_{a b} & =A_{b, a}-A_{a, b},
\end{aligned}
$$

where $A_{a}=\left(A_{t}, A_{\varphi}, 0,0\right)$ and the electromagnetic potentials $A_{t}$ and $A_{\varphi}$ are also functions of $r$ and $z$ only.

For the metric (1), the Einstein-Maxwell equations are equivalent to the system [44]

$$
\begin{gathered}
\nabla \cdot\left[r^{-2} f\left(\nabla A_{\varphi}-\mathcal{W} \nabla A_{t}\right]=0,\right. \\
\nabla \cdot\left[f^{-1} \nabla A_{t}+r^{-2} f \mathcal{W}\left(\nabla A_{\varphi}-\mathcal{W} \nabla A_{t}\right]=0,\right. \\
\nabla \cdot\left[r^{-2} f^{2} \nabla \mathcal{W}-2 r^{-2} f A_{t}\left(\nabla A_{\varphi}-\mathcal{W} \nabla A_{t}\right)\right]=0, \\
f \nabla^{2} f=\nabla f \cdot \nabla f-r^{-2} f^{4} \nabla \mathcal{W} \cdot \nabla \mathcal{W}+f \nabla A_{t} \cdot \nabla A_{t} \\
+r^{-2} f^{3}\left(\nabla A_{\varphi}-\mathcal{W} \nabla A_{t}\right) \cdot\left(\nabla A_{\varphi}-\mathcal{W} \nabla A_{t}\right), \\
\Lambda_{, r}=r\left(\Psi_{, r}^{2}-\Psi_{, z}^{2}\right)-\frac{1}{4 r}\left(\mathcal{W}_{, r}^{2}-\mathcal{W}_{, z}^{2}\right) e^{4 \Psi}-\frac{1}{2 r}\left(r^{2} e^{-2 \Psi}-\mathcal{W}^{2} e^{2 \Psi}\right)\left(A_{t, r}^{2}-A_{t, z}^{2}\right) \\
+\frac{1}{2 r}\left(A_{\varphi, r}^{2}-A_{\varphi, z}^{2}\right) e^{2 \Psi}-\frac{1}{r} \mathcal{W}\left(A_{\varphi, r} A_{t, r}-A_{\varphi, z} A_{t, z}\right) e^{2 \Psi}, \\
\Lambda_{, z}=2 r \Psi, r \\
\Psi_{, z}-\frac{1}{2 r} \mathcal{W}_{, r} \mathcal{W}_{, z} e^{4 \Psi}-\frac{1}{r}\left(r^{2} e^{-2 \Psi}-\mathcal{W}^{2} e^{2 \Psi}\right) A_{t, r} A_{t, z} \\
+\frac{1}{r} A_{\varphi, r} A_{\varphi, z} e^{2 \Psi}-\frac{1}{r} \mathcal{W}\left(A_{\varphi, r} A_{t, z}+A_{\varphi, z} A_{t, r}\right) e^{2 \Psi},
\end{gathered}
$$

where $\nabla$ is the standard differential operator in cylindrical coordinates and $f=e^{2 \Psi}$.

In order to obtain a solution of (2a) - (2b) representing a thin disk at $z=0$, we assume that the components of the metric tensor are continuous across the disk, but with first derivatives discontinuous on the plane $z=0$, with discontinuity functions

$$
b_{a b}=\left.g_{a b, z}\right|_{z=0^{+}}-\left.g_{a b, z}\right|_{z=0^{-}}=\left.2 g_{a b, z}\right|_{z=0^{+}} .
$$

Thus, by using the distributional approach [46-48] or the junction conditions on the extrinsic curvature of thin shells [49-51], the Einstein-Maxwell equations yield an energymomentum tensor $T_{a b}=T_{a b}^{\mathrm{elm}}+T_{a b}^{\mathrm{mat}}$, where $T_{a b}^{\mathrm{mat}}=Q_{a b} \delta(z)$, and a current density $J_{a}=j_{a} \delta(z)=-2 e^{2(\Psi-\Lambda)} A_{a, z} \delta(z)$, where $\delta(z)$ is the usual Dirac function with support on the disk. $T_{a b}^{\mathrm{elm}}$ is the electromagnetic tensor defined in Eq. (3a), $j_{a}$ is the current density on the plane $z=0$, and

$$
Q_{b}^{a}=\frac{1}{2}\left\{b^{a z} \delta_{b}^{z}-b^{z z} \delta_{b}^{a}+g^{a z} b_{b}^{z}-g^{z z} b_{b}^{a}+b_{c}^{c}\left(g^{z z} \delta_{b}^{a}-g^{a z} \delta_{b}^{z}\right)\right\}
$$

is the distributional energy-momentum tensor. The "true" surface energy-momentum tensor (SEMT) of the disk, $S_{a b}$, and the "true" surface current density, $j_{a}$, can be obtained through the relations

$$
\begin{aligned}
S_{a b} & =\int T_{a b}^{\mathrm{mat}} d s_{n}=e^{\Lambda-\Psi} Q_{a b}, \\
j_{a} & =\int J_{a} d s_{n}=e^{\Lambda-\Psi} j_{a},
\end{aligned}
$$


where $d s_{n}=\sqrt{g_{z z}} d z$ is the "physical measure" of length in the direction normal to the disk.

For the metric (1), the nonzero components of $S_{a}^{b}$ are

$$
\begin{aligned}
S_{0}^{0} & =\frac{e^{\Psi-\Lambda}}{r^{2}}\left[2 r^{2}\left(\Lambda,_{z}-2 \Psi,_{z}\right)-e^{4 \Psi} \mathcal{W} \mathcal{W},_{z}\right], \\
S_{1}^{0} & =-\frac{e^{\Psi-\Lambda}}{r^{2}}\left[4 r^{2} \mathcal{W} \Psi,_{z}+\left(r^{2}+\mathcal{W}^{2} e^{4 \Psi}\right) \mathcal{W},_{z}\right], \\
S_{0}^{1} & =\frac{e^{\Psi-\Lambda}}{r^{2}}\left[e^{4 \Psi} \mathcal{W},_{z}\right], \\
S_{1}^{1} & =\frac{e^{\Psi-\Lambda}}{r^{2}}\left[2 r^{2} \Lambda,_{z}+e^{4 \Psi} \mathcal{W} \mathcal{W},_{z}\right],
\end{aligned}
$$

and the nonzero components of the surface current density $j_{a}$ are

$$
\begin{aligned}
& j_{t}=-2 e^{\Psi-\Lambda} A_{t, z}, \\
& j_{\varphi}=-2 e^{\Psi-\Lambda} A_{\varphi, z},
\end{aligned}
$$

where all the quantities are evaluated at $z=0^{+}$.

These disks are essentially of infinite extension. Finite disks can be obtained introducing oblate spheroidal coordinates, which are naturally adapted to a disk source, and imposing appropriate boundary conditions. These solutions, in the vacuum and static case, correspond to the Morgan and Morgan solutions [3]. A more general class of solutions representating finite thin disks can be constructed using a method based on the use of conformal transformations and solving a boundaryvalue problem [4-6, 15, 30, 31].

Now, in order to analyze the matter content of the disks is necessary to compute the eigenvalues and eigenvectors of the energy-momentum tensor. The eigenvalue problem for the $\operatorname{SEMT~(7a)~-~(7d)~}$

$$
S_{b}^{a} \xi^{b}=\lambda \xi^{a}
$$

has the solutions

$$
\lambda_{ \pm}=\frac{1}{2}(T \pm \sqrt{D})
$$

where

$$
T=S_{0}^{0}+S_{1}^{1}, \quad D=\left(S_{1}^{1}-S_{0}^{0}\right)^{2}+4 S_{1}^{0} S_{0}^{1},
$$

and $\lambda_{r}=\lambda_{z}=0$. For the metric (1)

$$
\begin{aligned}
D & =4 \frac{e^{2(\Psi-\Lambda)}}{r^{2}}\left(4 r^{2} \Psi_{, z}^{2}-\mathcal{W}_{, z}^{2} e^{4 \Psi}\right)=A^{2}-B^{2}, \\
T & =4 e^{\Psi-\Lambda}\left(\Lambda_{, z}-\Psi_{, z}\right),
\end{aligned}
$$

where

$$
A=4 \Psi_{, z} e^{\Psi-\Lambda}, \quad B=\frac{2}{r} \mathcal{W}_{, z} e^{3 \Psi-\Lambda} .
$$

The corresponding eigenvectors are

$$
\begin{aligned}
\xi_{ \pm}^{a} & =\left(\xi_{ \pm}^{0}, \xi_{ \pm}^{1}, 0,0\right), \\
X^{a} & =e^{U-\Lambda}(0,0,1,0), \\
Y^{a} & =e^{U-\Lambda}(0,0,0,1),
\end{aligned}
$$

with

$$
g\left(\xi_{ \pm}, \xi_{ \pm}\right)=2 N_{ \pm} e^{2 \Psi}\left(\frac{\xi_{ \pm}^{0}}{S_{0}^{0}-S_{1}^{1} \pm \sqrt{D}}\right)^{2}
$$

where

$$
N_{ \pm}=\sqrt{D}(-\sqrt{D} \pm A) .
$$

We only consider the case when $D \geq 0$, so that the two eigenvalues $\lambda_{ \pm}$are real and different and the two eigenvectors are orthogonal, in such a way that one of them is timelike and the other is spacelike. Since $|A| \geq \sqrt{D}$, from (16) follows that when $A>0$ the negative sign corresponds to the timelike eigenvector while the positive sign to the spacelike eigenvector. When $A<0$ we have the opposite case. So the function $\Psi_{, z}$ determines the sign of the norm.

Let $V^{a}$ be the timelike eigenvector, $V_{a} V^{a}=-1$, and $W^{a}$ the spacelike eigenvector, $W_{a} W^{a}=1$. In terms of the orthonormal tetrad or comoving observer $\mathrm{e}_{\hat{a}}^{b}=\left\{V^{b}, W^{b}, X^{b}, Y^{b}\right\}$, the SEMT and the surface electric current density may be decomposed as

$$
\begin{aligned}
S_{a b} & =\varepsilon V_{a} V_{b}+p_{\varphi} W_{a} W_{b}, \\
j_{a} & =j^{\hat{0}} V_{a}+j^{\hat{1}} W_{a},
\end{aligned}
$$

where

$$
\varepsilon=-\lambda_{ \pm}, \quad p_{\varphi}=\lambda_{\mp},
$$

are, respectively, the surface energy density, the azimuthal pressure, and

$$
j^{\hat{0}}=-V^{a} j_{a}, \quad j^{\hat{1}}=W^{a} j_{a}
$$

are the surface electric charge density and the azimuthal current density of the disk measured by this observer. In (18) the sign is chosen according to which is the timelike eigenvector and which is the spacelike eigenvector. However, in order to satisfy the strong energy condition $\rho=\varepsilon+p_{\varphi} \geq 0$, where $\rho$ is the effective Newtonian density, we must choose $\xi_{-}$as the timelike eigenvector and $\xi_{+}$as the spacelike eigenvector. These condition characterizes a disk made of matter with the usual gravitational attractive property. Consequently $\Psi_{, z}$ must be taken positive. So we have

$$
\varepsilon=-\lambda_{-}, \quad p_{\varphi}=\lambda_{+},
$$

and

$$
\begin{aligned}
V^{0} & =\frac{v e^{-\Psi}}{\sqrt{-2 N_{-}}}\left(S_{0}^{0}-S_{1}^{1}-\sqrt{D}\right), \\
V^{1} & =\frac{2 v e^{-\Psi}}{\sqrt{-2 N_{-}}} S_{0}^{1},
\end{aligned}
$$

where $v= \pm$ so that the sign is chosen according to the causal character of the timelike eigenvector (observer's fourvelocity),

$$
\begin{aligned}
W^{0} & =\frac{2}{\sqrt{2 M}} S_{1}^{0}, \\
W^{1} & =\frac{1}{\sqrt{2 M}}\left(S_{1}^{1}-S_{0}^{0}+\sqrt{D}\right),
\end{aligned}
$$


where

$$
M=\sqrt{D}\left\{g_{11} \sqrt{D}+2 r \mathcal{W} B+\left(r^{2} e^{-2 \Psi}+\mathcal{W}^{2} e^{2 \Psi}\right) A\right\}
$$

\section{COUNTERROTATING CHARGED DUST DISKS}

We now consider, based on Refs. [15] and [30], the possibility that the SEMT $S^{a b}$ and the current density $j^{a}$ can be written as the superposition of two counterrotating charged fluids that circulate in opposite directions; that is, we assume

$$
\begin{aligned}
S^{a b} & =S_{+}^{a b}+S_{-}^{a b}, \\
j^{a} & =j_{+}^{a}+j_{-}^{a},
\end{aligned}
$$

where the quantities on the right-hand side are, respectively, the SEMT and the current density of the prograde and retrograde counterrotating fluids.

Let $U_{ \pm}^{a}=\left(U_{ \pm}^{0}, U_{ \pm}^{1}, 0,0\right)=U_{ \pm}^{0}\left(1, \omega_{ \pm}, 0,0\right)$ be the velocity vectors of the two counterrotating fluids, where $\omega_{ \pm}=U_{ \pm}^{1} / U_{ \pm}^{0}$ are the angular velocities of each stream. In order to do the decomposition (24a) and (24b) we project the velocity vectors onto the tetrad $\mathrm{e}_{\hat{a}}^{b}$, using the relations [52]

$$
U_{ \pm}^{\hat{a}}=\mathrm{e}^{\hat{a}}{ }_{b} U_{ \pm}^{b}, \quad U_{ \pm}^{a}=\mathrm{e}_{\hat{b}}^{a} U_{ \pm}^{\hat{b}} .
$$

In terms of the tetrad (14) we can write

$$
U_{ \pm}^{a}=\frac{V^{a}+v_{ \pm} W^{a}}{\sqrt{1-v_{ \pm}^{2}}},
$$

so that

$$
\begin{aligned}
V^{a} & =\frac{\sqrt{1-v_{-}^{2}} v_{+} U_{-}^{a}-\sqrt{1-v_{+}^{2}} v_{-} U_{+}^{a}}{v_{+}-v_{-}}, \\
W^{a} & =\frac{\sqrt{1-v_{+}^{2}} U_{+}^{a}-\sqrt{1-v_{-}^{2}} U_{-}^{a}}{v_{+}-v_{-}},
\end{aligned}
$$

where $v_{ \pm}=U_{ \pm}^{\hat{1}} / U_{ \pm}^{\hat{0}}$ are the tangential velocities of the streams with respect to the tetrad.

Another quantity related with the counterrotating motion is the specific angular momentum of a particle rotating at a radius $r$, defined as $h_{ \pm}=g_{\varphi a} U_{ \pm}^{a}$. This quantity can be used to analyze the stability of circular orbits of test particles against radial perturbations. The condition of stability,

$$
\frac{d\left(h^{2}\right)}{d r}>0
$$

is an extension of Rayleigh criteria of stability of a fluid in rest in a gravitational field [53]. For an analysis of the stability of a rotating fluid taking into account the collective behavior of the particles see for example Refs. [19, 54].
Substituting (27a) and (27b) in (17a) we obtain

$$
\begin{aligned}
S^{a b} & =\frac{F\left(v_{-}, v_{-}\right)\left(1-v_{+}^{2}\right) U_{+}^{a} U_{+}^{b}}{\left(v_{+}-v_{-}\right)^{2}} \\
& +\frac{F\left(v_{+}, v_{+}\right)\left(1-v_{-}^{2}\right) U_{-}^{a} U_{-}^{b}}{\left(v_{+}-v_{-}\right)^{2}} \\
& -\frac{F\left(v_{+}, v_{-}\right)\left(1-v_{+}^{2}\right)^{\frac{1}{2}}\left(1-v_{-}^{2}\right)^{\frac{1}{2}}\left(U_{+}^{a} U_{-}^{b}+U_{-}^{a} U_{+}^{b}\right)}{\left(v_{+}-v_{-}\right)^{2}}
\end{aligned}
$$

where

$$
F\left(v_{1}, v_{2}\right)=\varepsilon v_{1} v_{2}+p_{\varphi} .
$$

Clearly, in order to cast the SEMT in the form (24a), the mixed term must be absent and therefore the counterrotating tangential velocities must satisfy the following constraint

$$
F\left(v_{+}, v_{-}\right)=\varepsilon v_{+} v_{-}+p_{\varphi}=0
$$

where we assume that $\left|v_{ \pm}\right| \neq 1$.

Then, assuming a given choice for the tangential velocities in agreement with the above relation, we can write the SEMT as (24a) with

$$
S_{ \pm}^{a b}=\varepsilon_{ \pm} U_{ \pm}^{a} U_{ \pm}^{b}
$$

so that we have two counterrotating dust fluids with surface energy densities, measured in the coordinates frames, given by

$$
\varepsilon_{ \pm}=\left[\frac{1-v_{ \pm}^{2}}{v_{\mp}-v_{ \pm}}\right] \varepsilon v_{\mp},
$$

Thus the SEMT $S^{a b}$ can be written as the superposition of two counterrotating dust fluids if, and only if, the constraint (30) admits a solution such that $v_{+} \neq v_{-}$.

Similarly, substituting (27a) and (27b) in (17b) we can write the current density as (24b) with

$$
j_{ \pm}^{a}=\sigma_{ \pm} U_{ \pm}^{a}
$$

where $\sigma_{ \pm}$are the surface electric charge densities, measured in the coordinates frames,

$$
\sigma_{ \pm}=\left[\frac{\sqrt{1-v_{ \pm}^{2}}}{v_{ \pm}-v_{\mp}}\right]\left(j^{\hat{1}}-j^{\hat{0}} v_{\mp}\right) .
$$

Thus, we have a disk makes of two counterrotating charged dust fluids with surface energy densities given by (32), and surface electric charge densities given by (34).

As we can see from Eqs. (26), (32) and (34), all the main physical quantities associated with the CRM depend on the counterrotating tangential velocities $v_{ \pm}$. However, the constraint (30) does not determine $v_{ \pm}$uniquely so that we need to impose some additional requirement in order to obtain a complete determination of the tangential velocities leading to a well defined CRM. 
A possibility, commonly assumed [25, 38], is to take the two counterrotating streams as circulating along electrogeodesics. Now, if the electrogeodesic equation admits solutions corresponding to circular orbits, we can write this equation as

$$
\frac{1}{2} \varepsilon_{ \pm} g_{a b, r} U_{ \pm}^{a} U_{ \pm}^{b}=-\sigma_{ \pm} F_{r a} U_{ \pm}^{a} .
$$

In terms of $\omega_{ \pm}$we obtain

$\frac{1}{2} \varepsilon_{ \pm}\left(U_{ \pm}^{0}\right)^{2}\left(g_{11, r} \omega_{ \pm}^{2}+2 g_{01, r} \omega_{ \pm}+g_{00, r}\right)=-\sigma_{ \pm} U_{ \pm}^{0}\left(A_{t, r}+A_{\varphi, r} \omega_{ \pm}\right)$.

(36) and substituting (37a) and (37b) in (36) we find

From (24a), (24b), (31), and (33) we have

$$
\begin{aligned}
\sigma_{ \pm} U_{ \pm}^{0} & =\frac{j^{1}-\omega_{\mp} j^{0}}{\omega_{ \pm}-\omega_{\mp}}, \\
\varepsilon_{ \pm}\left(U_{ \pm}^{0}\right)^{2} & =\frac{S^{01}-\omega_{\mp} S^{00}}{\omega_{ \pm}-\omega_{\mp}} \\
\omega_{\mp} & =\frac{S^{11}-\omega_{ \pm} S^{01}}{S^{01}-\omega_{ \pm} S^{00}},
\end{aligned}
$$

$$
\frac{1}{2}\left(S^{01}-\omega_{\mp} S^{00}\right)\left(g_{11, r} \omega_{ \pm}^{2}+2 g_{01, r} \omega_{ \pm}+g_{00, r}\right)=-\left(j^{1}-\omega_{\mp} j^{0}\right)\left(A_{t, r}+A_{\varphi, r} \omega_{ \pm}\right),
$$

and using (37c) we obtain

$$
\frac{1}{2}\left[\left(S^{01}\right)^{2}-S^{00} S^{11}\right]\left(g_{11, r} \omega_{ \pm}^{2}+2 g_{01, r} \omega_{ \pm}+g_{00, r}\right)=-\left[S^{01} j^{1}-S^{11} j^{0}+\omega_{ \pm}\left(S^{01} j^{0}-S^{00} j^{1}\right)\right]\left(A_{t, r}+A_{\varphi, r} \omega_{ \pm}\right) .
$$

Therefore we conclude that

$$
\omega_{ \pm}=\frac{-T_{2} \pm \sqrt{T_{2}^{2}-T_{1} T_{3}}}{T_{1}}
$$

with

$$
\begin{aligned}
& T_{1}=g_{11, r}+2 A_{\varphi, r} \frac{j^{0} S^{01}-j^{1} S^{00}}{S^{01} S^{01}-S^{00} S^{11}}, \\
& T_{2}=g_{01, r}+A_{t, r} \frac{j^{0} S^{01}-j^{1} S^{00}}{S^{01} S^{01}-S^{00} S^{11}}+A_{\varphi, r} \frac{j^{1} S^{01}-j^{0} S^{11}}{S^{01} S^{01}-S^{00} S^{11}}, \\
& T_{3}=g_{00, r}+2 A_{t, r} \frac{j^{1} S^{01}-j^{0} S^{11}}{S^{01} S^{01}-S^{00} S^{11}} .
\end{aligned}
$$

On the other hand, in terms of $\omega_{ \pm}$we get

$$
v_{ \pm}=-\left[\frac{W_{0}+W_{1} \omega_{ \pm}}{V_{0}+V_{1} \omega_{ \pm}}\right]
$$

and so, by using (40), we have that

$$
v_{+} v_{-}=\frac{T_{1} W_{0}^{2}-2 T_{2} W_{0} W_{1}+T_{3} W_{1}^{2}}{T_{1} V_{0}^{2}-2 T_{2} V_{0} V_{1}+T_{3} V_{1}^{2}},
$$

so that, using $(17 \mathrm{a})$, we get

$$
\begin{aligned}
F\left(v_{+}, v_{-}\right)= & \frac{32 e^{4(\Psi-\Lambda)} \Lambda_{, z}^{2}\left(r^{2} \Lambda_{, z} \sqrt{D}+4 r^{2} \Lambda_{, z} \Psi_{, z} e^{\Psi-\Lambda}-\mathcal{W}_{, z}^{2} e^{5 \Psi-\Lambda}\right)}{r^{3}(A+\sqrt{D}) p_{\varphi}\left(S^{01} S^{01}-S^{00} S^{11}\right)\left(T_{1} V_{0}^{2}-2 T_{2} V_{0} V_{1}+T_{3} V_{1}^{2}\right)} \\
& \times\left[\Lambda_{, z}-2 r \Psi_{, r} \Psi_{, z}+\frac{1}{2 r} \mathcal{W}_{, r} \mathcal{W}_{, z} e^{4 \Psi}+\frac{1}{r}\left(r^{2} e^{-2 \Psi}-\mathcal{W}^{2} e^{2 \Psi}\right) A_{t, r} A_{t, z}\right. \\
& \left.-\frac{1}{r} A_{\varphi, r} A_{\varphi, z} e^{2 \Psi}+\frac{1}{r} \mathcal{W}\left(A_{\varphi, r} A_{t, z}+A_{\varphi, z} A_{t, r}\right) e^{2 \Psi}\right]
\end{aligned}
$$

Finally, using the Einstein-Maxwell equation (4f) follows immediately that $F\left(v_{+}, v_{-}\right)$vanishes and therefore the electro- 
geodesic velocities satisfy the constraint (30) and so, if the electrogeodesic equation admits solutions corresponding to circular orbits, we have a well defined CRM.

Another possibility is to take the two counterrotating fluids not circulating along electrogeodesics but with equal and opposite tangential velocities,

$$
v_{ \pm}= \pm v= \pm \sqrt{p_{\varphi} / \varepsilon}
$$

This choice, that imply the existence of additional interactions between the two streams (e.g. collisions), leads to a complete determination of the velocity vectors. However, this can be made only when $0 \leq\left|p_{\varphi} / \varepsilon\right| \leq 1$. In the general case, the two counterrotating streams circulate with different velocities and we can write (30) as

$$
v_{+} v_{-}=-\frac{p_{\varphi}}{\varepsilon} .
$$

However, this relation does not determine completely the tangential velocities, and therefore the CRM is undetermined.

In summary, the counterrotating tangential velocities can be explicitly determined only if we assume some additional relationship between them, like the equal and opposite condition or the electro-geodesic condition. Now, can happen that the obtained solutions do not satisfy any of these two conditions. That is, the counterrotating velocities are, in general, not completely determined by the constraint (30). Thus, the
CRM is in general undetermined since the counterrotaing energy densities and pressures can not be explicitly written without a knowledge of the counterrotating tangential velocities.

\section{DISKS FROM A KERR-NEWMAN SOLUTION}

As an example of the above presented formalism, we consider the thin disk models obtained by means of the "displace, cut and reflect" method applied to the well known KerrNewman solution, which can be written as

$$
\begin{aligned}
\Psi & =\frac{1}{2} \ln \left[\frac{a^{2} x^{2}+b^{2} y^{2}-c^{2}}{\left(a x+c^{2}\right)^{2}+b^{2} y^{2}}\right], \\
\Lambda & =\frac{1}{2} \ln \left[\frac{a^{2} x^{2}+b^{2} y^{2}-c^{2}}{a^{2}\left(x^{2}-y^{2}\right)}\right], \\
\mathcal{W} & =\frac{c^{2} k b\left(1-y^{2}\right)\left(2 a x+1+c^{2}\right)}{a\left(a^{2} x^{2}+b^{2} y^{2}-c^{2}\right)}, \\
A_{t} & =\frac{c \sqrt{2\left(c^{2}-1\right)}\left(a x+c^{2}\right)}{\left(a x+c^{2}\right)^{2}+b^{2} y^{2}}, \\
A_{\varphi} & =-k \frac{b}{a}\left(1-y^{2}\right) A_{t},
\end{aligned}
$$

where $a^{2}+b^{2}=c^{2} \geq 1$, with

$$
a=\frac{k}{m(1-q q *)}, \quad b=\frac{L}{m\left(1-q q^{*}\right)}, \quad c=\frac{1}{\sqrt{1-q q^{*}}}, \quad k=\sqrt{m^{2}-L^{2}-e^{2}}, \quad|q|=\frac{e}{m},
$$

where $m, L$ and $e$ are the mass, angular momentum and electric charge parameters of the Kerr-Newman black hole, respectively. Note that $c$ is the parameter that controls the electromagnetic field. The prolate spheroidal coordinates, $x$ and $y$, are related with the Weyl coordinates by

$$
r^{2}=k^{2}\left(x^{2}-1\right)\left(1-y^{2}\right), \quad z+z_{0}=k x y,
$$

where $1 \leq x \leq \infty, 0 \leq y \leq 1$, and $k$ is an arbitrary constant. Note that we have displaced the origin of the $z$ axis in $z_{0}$. This solution can be generated, in these coordinates, using the well-known complex potential formalism proposed by Ernst [44] from the Kerr vacuum solution [1]. When $c=1$ this solution reduces to the Kerr vacuum solution.

Let be $\tilde{D}=k^{2} D, \tilde{T}=k T$, and $\tilde{j}_{t}=k j_{t}$, therefore

$$
\begin{aligned}
\tilde{T}= & \frac{4 c^{2} a \bar{y}\left\{2 \bar{x}\left(1-\bar{y}^{2}\right)\left(\bar{x}^{2}+2 a \bar{x}+c^{2}\right)-\left(\bar{x}^{2}-\bar{y}^{2}\right)\left[a\left(\bar{x}^{2}+1\right)+\bar{x}\left(1+c^{2}\right)\right]\right\}}{\left(\bar{x}^{2}-\bar{y}^{2}\right)^{3 / 2}\left[\left(a \bar{x}+c^{2}\right)^{2}+b^{2} \bar{y}^{2}\right]^{3 / 2}}, \\
\tilde{D}= & \frac{16 c^{4} a^{2} \bar{y}^{2}\left\{\left[a\left(\bar{x}^{2}+1\right)+\bar{x}\left(1+c^{2}\right)\right]^{2}-b^{2} \tilde{r}^{2}\right\}}{\left(\bar{x}^{2}-\bar{y}^{2}\right)\left[\left(a \bar{x}+c^{2}\right)^{2}+b^{2} \bar{y}^{2}\right]^{3}} \\
\tilde{j}_{t}= & \frac{2 c \sqrt{2\left(c^{2}-1\right)} a \bar{y}\left\{-b^{2} \bar{y}^{2}\left(3 a \bar{x}^{2}+2 \bar{x} c^{2}-a\right)+\left(a \bar{x}+c^{2}\right)\left(a^{2} \bar{x}^{3}+a c^{2} \bar{x}^{2}\right.\right.}{\left(\bar{x}^{2}-\bar{y}^{2}\right)^{1 / 2}\left[\left(a \bar{x}+c^{2}\right)^{2}+b^{2} \bar{y}^{2}\right]^{5 / 2}} \\
& \left.\left.-a^{2} \bar{x}+2 b^{2} \bar{x}-a c^{2}\right)\right\} \\
j_{\varphi}= & -\frac{2 c \sqrt{2\left(c^{2}-1\right)} b \bar{y}\left(1-\bar{y}^{2}\right)\left\{-a b^{2} \bar{y}^{2}\left(\bar{x}^{2}-1\right)+\left(a \bar{x}+c^{2}\right)\left(3 a^{2} \bar{x}^{3}\right.\right.}{\left(\bar{x}^{2}-\bar{y}^{2}\right)^{1 / 2}\left[\left(a \bar{x}+c^{2}\right)^{2}+b^{2} \bar{y}^{2}\right]^{5 / 2}} \\
& \left.\left.+5 a c^{2} \bar{x}^{2}-a^{2} \bar{x}+2 b^{2} \bar{x}+2 c^{4} \bar{x}-a c^{2}\right)\right\} .
\end{aligned}
$$


In the above expressions $\bar{x}$ and $\bar{y}$ are given by

$$
\begin{aligned}
& 2 \bar{x}=\sqrt{\tilde{r}^{2}+(\alpha+1)^{2}}+\sqrt{\tilde{r}^{2}+(\alpha-1)^{2}}, \\
& 2 \bar{y}=\sqrt{\tilde{r}^{2}+(\alpha+1)^{2}}-\sqrt{\tilde{r}^{2}+(\alpha-1)^{2}},
\end{aligned}
$$

where $\tilde{r}=r / k$ and $\alpha=z_{0} / k$, with $\alpha>1$.
Now, in order to analyze the behavior of $D$, is enough to consider the expression

$$
\tilde{D}_{0}=\left[a\left(\bar{x}^{2}+1\right)+\bar{x}\left(1+c^{2}\right)\right]^{2}-b^{2} \tilde{r}^{2}
$$

that can be written as

$$
\begin{aligned}
\tilde{D}_{0}= & a\left(1+c^{2}\right) R_{+}[\alpha(\alpha-1)+2+\tilde{r}]+a\left(1+c^{2}\right) R_{-}[\alpha(\alpha+1)+2+\tilde{r}] \\
& +\frac{1}{2} R_{+} R_{-}\left[\left(c^{2}+1\right)^{2}+a^{2}\left(\tilde{r}^{2}+\alpha^{2}+3\right)\right]+\frac{1}{2}\left[\left(c^{2}+1\right)^{2}+5 a^{2}\right] \\
& +\frac{1}{2} \tilde{r}^{2}\left[c^{4}+1+a^{2}\left(\tilde{r}^{2}+2 \alpha^{2}+6\right)\right]+\frac{1}{2} \alpha^{2}\left[\left(c^{2}+1\right)^{2}+a^{2}\left(\alpha^{2}+2\right)\right],
\end{aligned}
$$

where $R_{ \pm}=\sqrt{\tilde{r}^{2}+(\alpha \pm 1)^{2}}$. Since $\alpha(\alpha \mp 1)+2>0$ for any $\alpha$, from (53) follows that $D$ always is a positive quantity for Kerr-Newman fields and therefore the eigenvalues of the energy-momentum tensor are always real quantities. So we conclude that these disks can be interpreted, for all the values of parameters, as a matter distribution with currents and purely azimuthal pressure and without heat flow.

We can see also that for the vacuum case, when $c=1, D$ is everywhere positive. These disks, obtained from the Kerr vacum solution, were previously considered by González and Letelier in the reference [17]. In this previous work, due to a mistake in the computation of the expressions for $\tilde{T}$ and $\tilde{D}$, was concluded that the energy-momentum tensor could present complex eigenvalues for some values of the parameters. As we can see from the expressions presented here, this is not correct and, in all the cases, we have a matter distributions with purely azimuthal pressure and without heat flow for all the values of parameters.

In order to study the behavior of the main physical quantities associated with the disks, we perform a graphical analysis of them for disks with $\alpha=2, b=0.2$ and $c=1.0,1.5,2.0,2.5$, and 3.5, as functions of $\tilde{r}$. For these values of the parameters we find that $\Psi_{, z}$ is a positive quantity in agreement with the strong energy condition. Therefore, $\varepsilon=-\lambda_{-}$and $p_{\varphi}=\lambda_{+}$. However, one also finds values of the parameter for which $\Psi_{, z}$ takes negative values. Furthermore, we take $v=-1$ in order to $V^{a}$ be a future-oriented timelike vector.

In Fig. 1 we show the surface energy density $\tilde{\varepsilon}$ and the azimuthal pressure $\tilde{p}_{\varphi}$. We see that the energy density presents a maximum at $\tilde{r}=0$ and then decreases rapidly with $\tilde{r}$, being always a positive quantity in agreement with the weak energy condition. We also see that the presence of electromagnetic field decreases the energy density at the central region of the disk and later increases it. We can observe that the pressure increases rapidly as one moves away from the disk center, reaches a maximum and later decreases rapidly. We also observe that the electromagnetic field decreases the pressure everywhere on the disk.

The electric charge density $\tilde{j}_{t}$ and the azimuthal current density $j_{\varphi}$, measured in the coordinates frame, are represented in Fig. 2, whereas the electric charge density $\tilde{j}^{\hat{0}}$ and the azimuthal current density $\tilde{j}^{\hat{j}}$, measured by the comoving observer, are represented in Fig. 3. We observe that the electric charge density has a similar behavior to the energy and that the current density have a similar behavior to the pressure which is consistent with the fact that the mass is more concentrated in the disks center. We also computed this functions for other values of the parameters and, in all the cases, we found the same behavior.

We now consider the CRM for the same values of the parameters. We first consider the two counterrotating streams circulating along electrogeodesics. In Fig. 4 we plot the tangential velocity curves, $v_{+}$and $v_{-}$. We see that these velocities are always less than the light velocity. We also see that the inclusion of the electromagnetic field make less relativistic these disks. In Fig. 5 we have drawn the specific angular momenta $h_{+}^{2}$ and $h_{-}^{2}$ for the same values of the parameters. We see that the presence of electromagnetic field can make unstable these orbits against radial perturbations. Thus the CRM cannot apply for $c=6$ (bottom curve). In Fig. 6 we have plotted the surface energy densities $\tilde{\varepsilon}_{+}$and $\tilde{\varepsilon}_{-}$. We see that these quantities have a similar behavior to the energy density $\tilde{\varepsilon}$. In Fig. 7 , we plotted the surface electric charge densities $\tilde{\sigma}_{+}$and $\tilde{\sigma}_{-}$. We find that these quantities have also a similar behavior to $\tilde{\varepsilon}_{ \pm}$.

The Figs. 4 - 7 show that the two fluids are continuous in $r$ which implies to have two particles in counterrotating movement in the same point in spacetime. So this model could be possible when the distance between streams (or between the counterrotating particles) were very small in comparing with the length $r$ so that we can consider, in principle, the fluids continuous like is the case of counterrotating gas disks present in disk galaxies.

Finally, in the case when the two fluids move with equal and opposite tangential velocities (non-electrogeodesic motion) we find that the physical quantities have a similar behavior to the previous one. 
$\tilde{\varepsilon}$

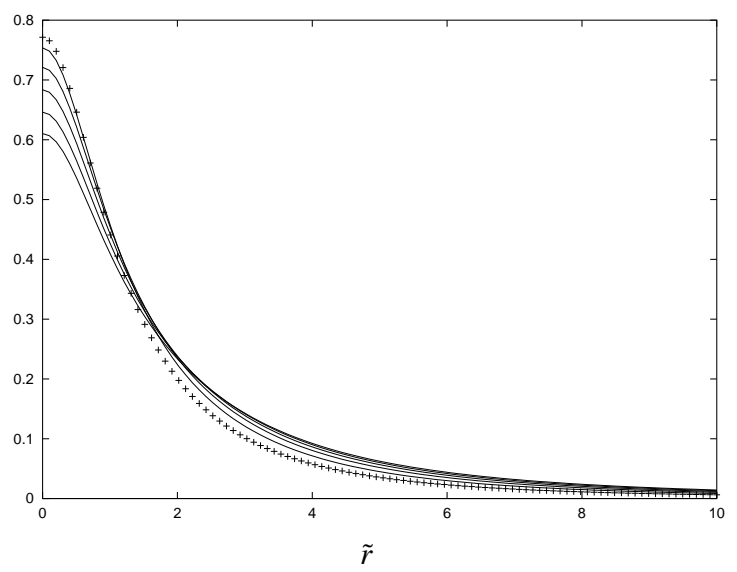

(a)

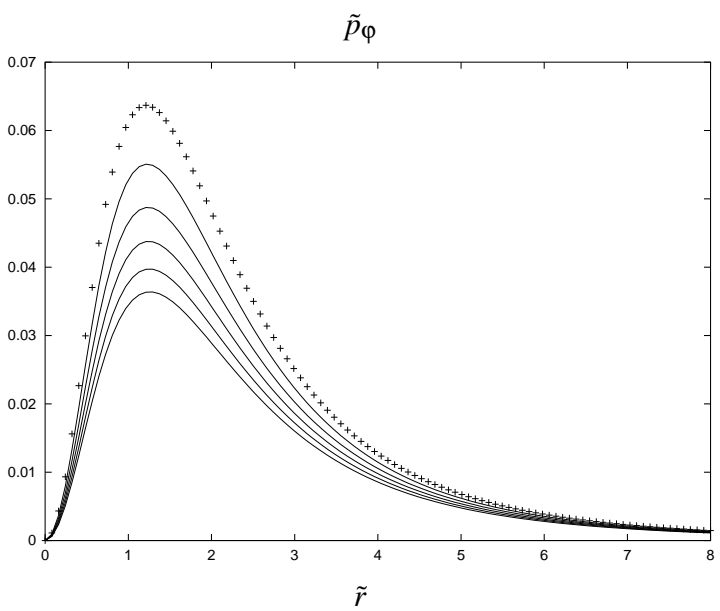

$(b)$

FIG. 1: $(a)$ The surface energy density $\tilde{\varepsilon}$ and $(b)$ the azimuthal pressure $\tilde{p}_{\varphi}$ for Kerr-Newman disks with $\alpha=2, b=0.2$ and $c=1.0$ (curves with crosses), 1.5, 2.0, 2.5, 3.0, and 3.5 (bottom curves), as functions of $\tilde{r}$.

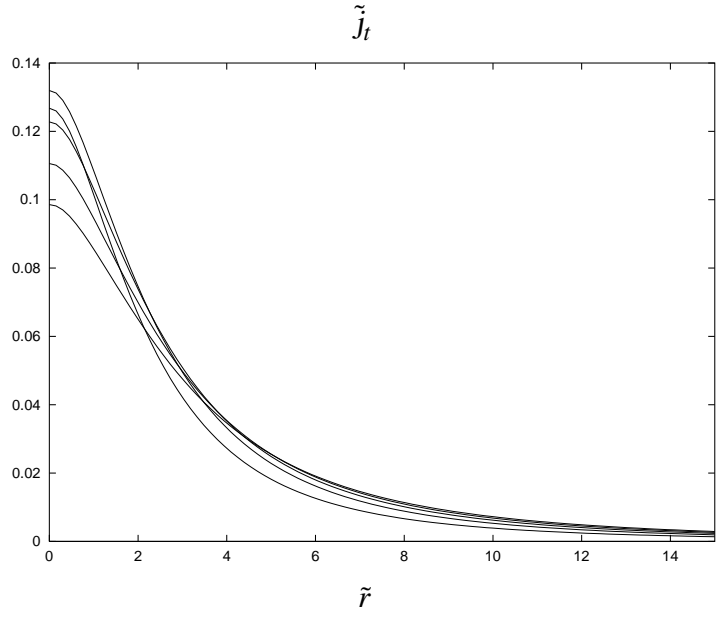

(a)

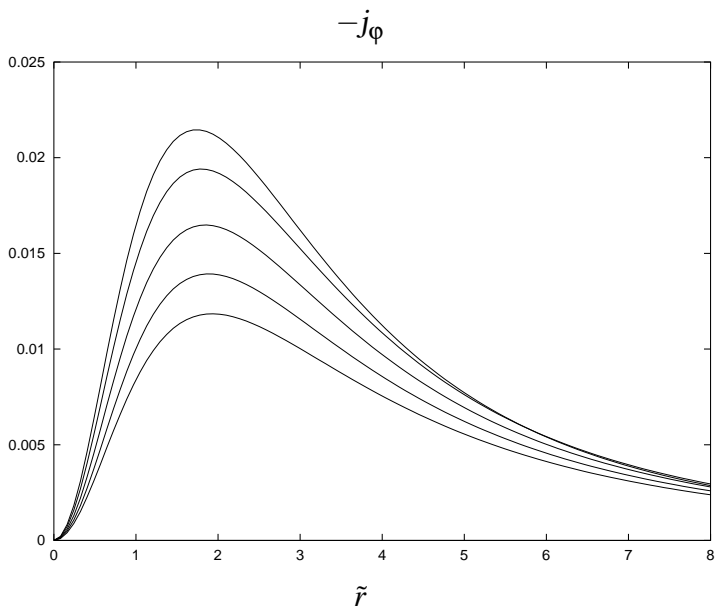

(b)

FIG. 2: $(a)$ The surface electric charge density $\tilde{j}_{t}$ and $(b)$ the azimuthal current density $j_{\varphi}$ for Kerr-Newman disks with $\alpha=2, b=0.2$ and $c=1.0$ (axis $\tilde{r}$ ), 1.5 (top curves), 2.0, 2.5, 3.0, and 3.5 (bottom curves), as functions of $\tilde{r}$.

\section{DISCUSSION}

We presented a detailed analysis of the energy-momentum tensor and the surface current density for electrovacuum stationary axially symmetric relativistic thin disks of infinite extension without radial stress, in the case when the energymomentum tensor of the disks can be diagonalized, so that there is not heat flow. The surface energy-momentum tensor and the surface current density were expressed in terms of the comoving tetrad and explicit expressions were obtained for the kinematical and dynamical variables that characterize the disks. That is, we obtained expressions for the velocity vector of the disks, as well for the energy density, azimuthal pressure, electric charge density and azimuthal current density.
We also presented in this paper the stationary generalization of the Counterrotating Model (CRM) for electrovacuum thin disks previously analyzed for the static case in [28, 30]. Thus then, we were able to obtain explicit expressions for all the quantities involved in the CRM that are fulfilled when do not exists heat flow and when we do not have radial pressure. We considered both counter rotation with equal and opposite velocities and counter rotation along electrogeodesics and, in both of the cases, we found the necessary conditions for the existence of a well defined CRM.

A general constraint over the counterrotating tangential velocities was obtained, needed to cast the surface energymomentum tensor of the disk in such a way that can be interpreted as the superposition of two counterrotating dust fluids. 


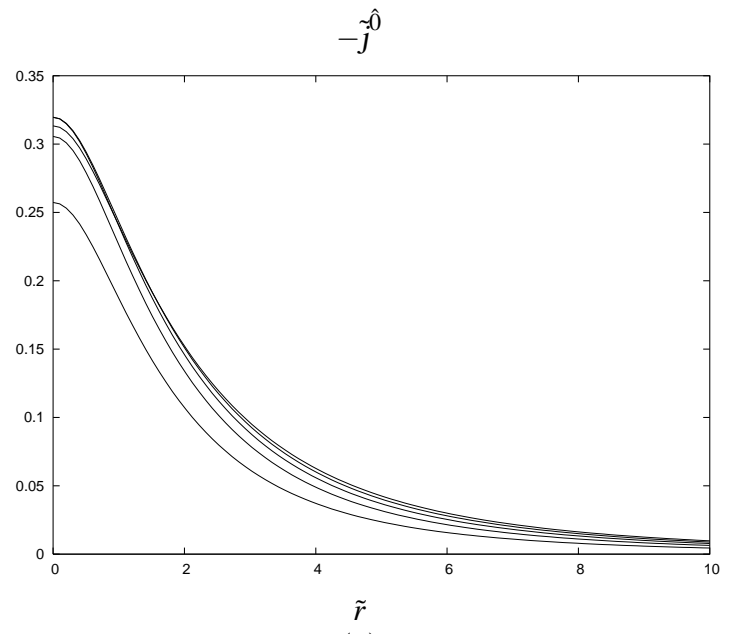

(a)

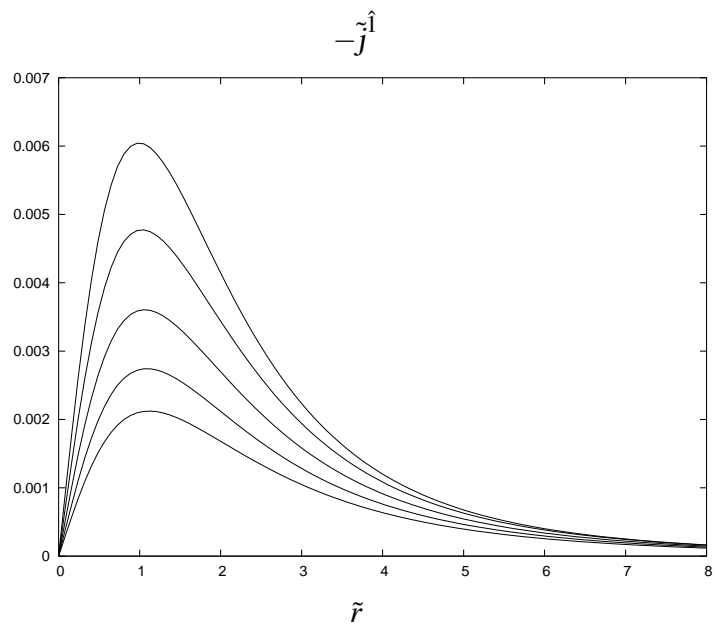

(b)

FIG. 3: For Kerr-Newman disks we plot, as function of $\tilde{r},(a) \hat{j}^{\hat{0}}$ with $\alpha=2, b=0.2$, and $c=1.0$ (axis $\tilde{r}$ ), 1.5 (bottom curve, away from the center of disk), 2.0, 2.5, 3.0, and 3.5 (top curve, away from the center of disk), and (b) $j^{\hat{1}}$ also with $\alpha=2, b=0.2$, and $c=1.0$ (axis $\tilde{r}$ ), 1.5 (top curve), 2.0, 2.5, 3.0, and 3.5 (bottom curve).

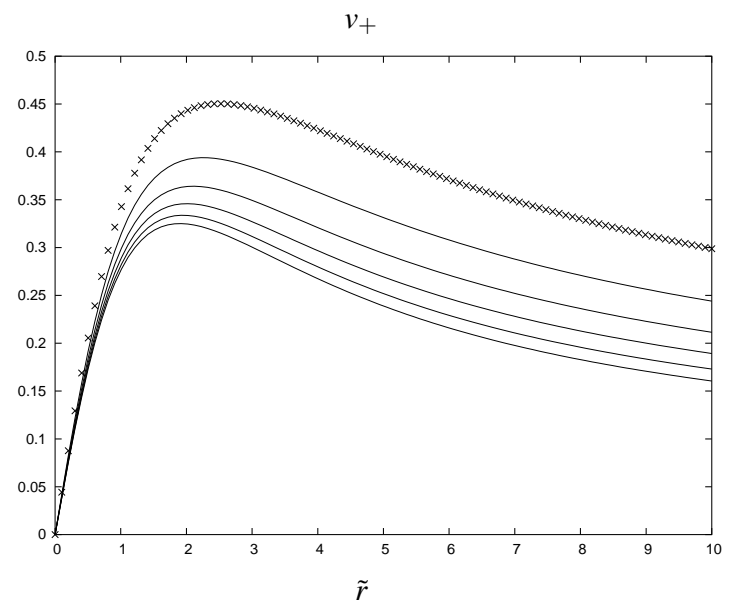

(a)

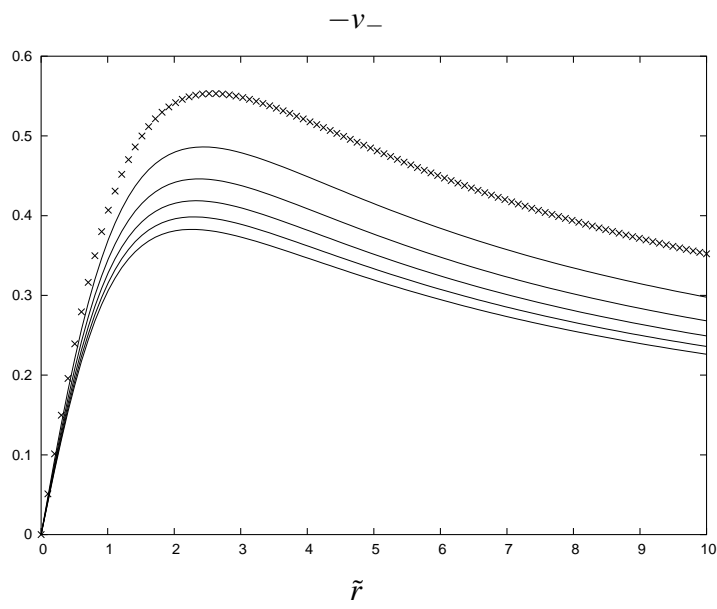

(b)

FIG. 4: The tangential velocities $(a) v_{+}$and $(b) v_{-}$for electrogeodesic Kerr-Newman disks with $\alpha=2, b=0.2$ and $c=1.0$ (curves with crosses), 1.5, 2.0, 2.5, 3.0, and 3.5 (bottom curves), as functions of $\tilde{r}$.

The constraint obtained is the generalization of the obtained for the vacuum case in [17], for disks without radial pressure or heat flow, where we only consider counterrotating fluids circulating along geodesics. We also found that, in general, there is not possible to take the two counterrotating tangential velocities as equal and opposite neither take the two counterrotating fluids as circulating along geodesics.

A simple family of models of counterrotating charged disks based on the Kerr-Newman solution were considered where we obtain some disks with a CRM well behaved. We also find that the disks constructed from the Kerr-Newman solution can be interpreted, for all the values of parameters, as a matter distribution with currents and purely azimuthal pressure and without heat flow. We obtain, for all the values of parameters, counterrotating Kerr-Newman disks that are in agreement with all the energy conditions. Finally, the generalization of these models to the case of electrovacuum stationary axially symmetric solutions where the energy-momentum tensor of the disk can to present complex eigenvalues for some values of the parameters, the stability of counterrotating fluids taking into account the collective behavior of the particles, and a thermodynamic analysis of the disks, will be considered in future works. 


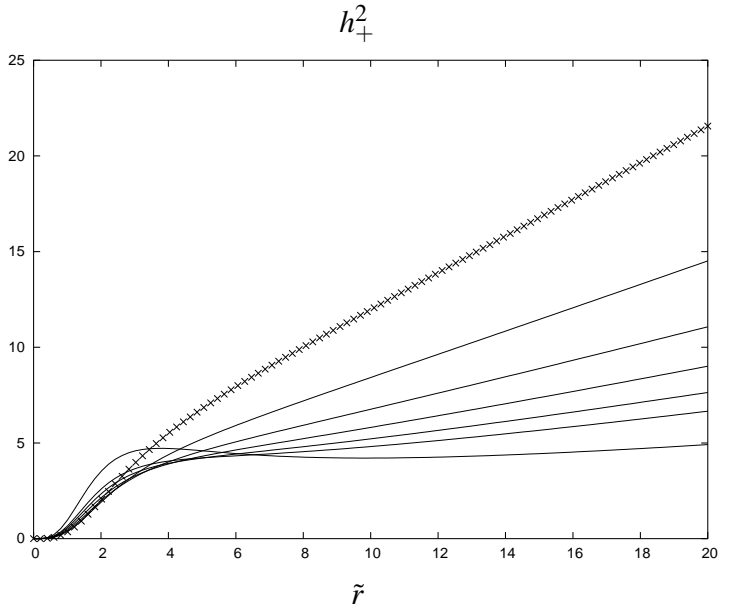

(a)

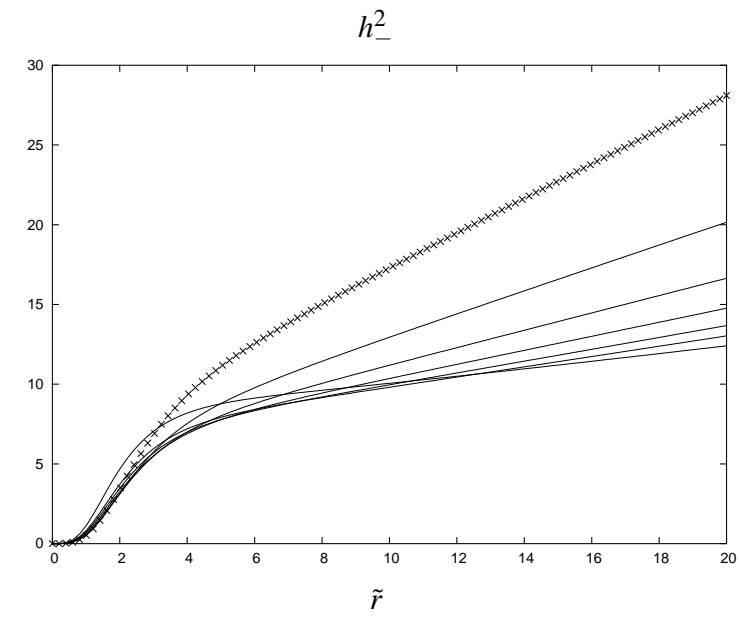

$(b)$

FIG. 5: The specific angular momenta $(a) h_{+}^{2}$ and (b) $h_{-}^{2}$ for electrogeodesic Kerr-Newman disks with $\alpha=2, b=0.2$ and $c=1.0$ (curves with crosses), 1.5, 2.0, 2.5, 3.0, 3.5, and 6.0 (bottom curves), as functions of $\tilde{r}$.

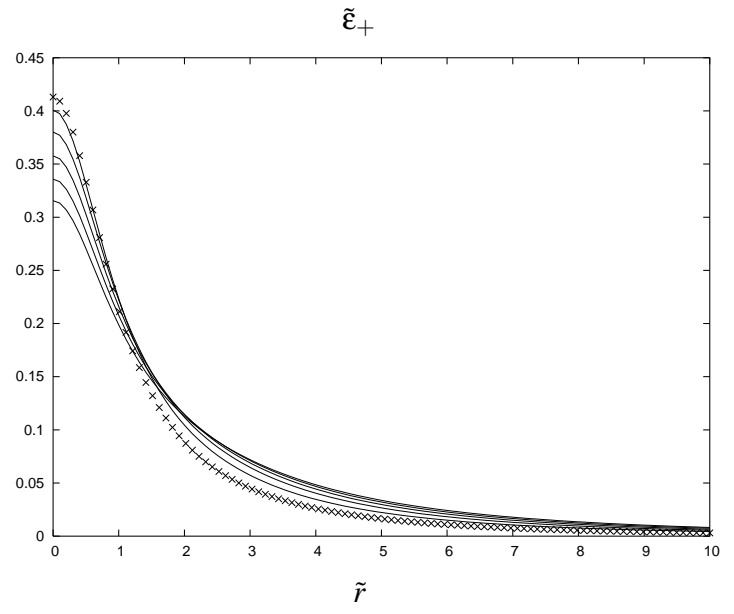

(a)

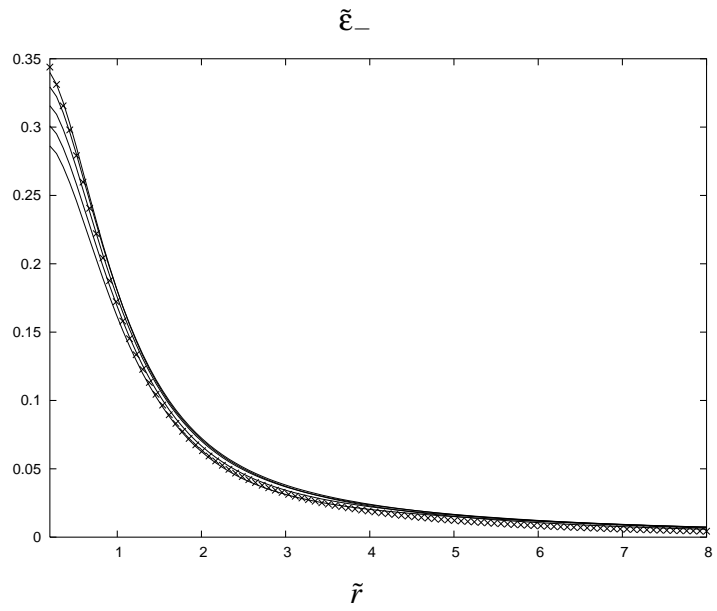

(b)

FIG. 6: The surface energy densities $(a) \tilde{\varepsilon}_{+}$and $(b) \tilde{\varepsilon}_{-}$for electrogeodesic Kerr-Newman disks with $\alpha=2, b=0.2$ and $c=1.0$ (curves with crosses), 1.5, 2.0, 2.5, 3.0, and 3.5 (bottom curves), as functions of $\tilde{r}$.

\section{Acknowledgments}

The authors want to thank the financial support from COLCIENCIAS, Colombia.

[1] D. Kramer, H. Stephani, E. Herlt, and M. McCallum, Exact Solutions of Einsteins's Field Equations (Cambridge University Press, Cambridge, England, 1980).

[2] W. A. Bonnor and A. Sackfield, Commun. Math. Phys. 8, 338 (1968).

[3] T. Morgan and L. Morgan, Phys. Rev. 183, 1097 (1969).

[4] L. Morgan and T. Morgan, Phys. Rev. D 2, 2756 (1970).
[5] A. Chamorro, R. Gregory, and J. M. Stewart, Proc. R. Soc. London A 413, 251 (1987).

[6] G. A. González and P. S. Letelier, Class. Quantum Grav. 16, 479 (1999).

[7] D. Lynden-Bell and S. Pineault, Mon. Not. R. Astron. Soc. 185, 679 (1978).

[8] P. S. Letelier and S. R. Oliveira, J. Math. Phys. 28, 165 (1987). 


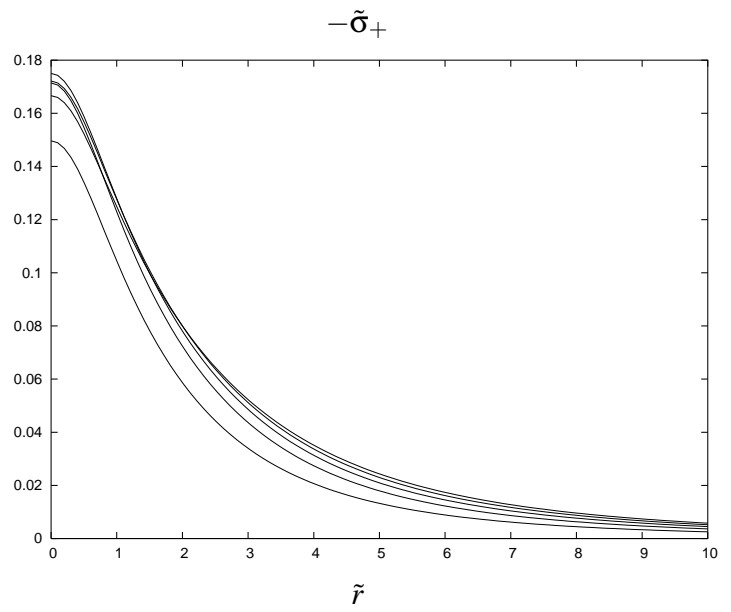

(a)

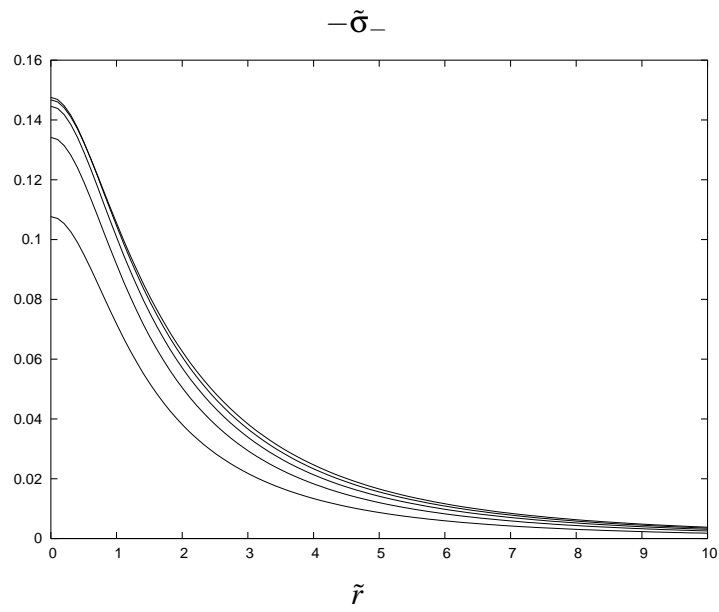

$(b)$

FIG. 7: The surface electric charge densities $(a) \tilde{\sigma}_{+}$and $(b) \tilde{\sigma}_{-}$for electrogeodesic Kerr-Newman disks with $\alpha=2, b=0.2$ and $c=1.0$ (axis $\tilde{r}), 1.5,2.0,2.5,3.0$, and 3.5 (top curves away from the center of the disk), as functions of $\tilde{r}$.

[9] J. P. S. Lemos, Class. Quantum Grav. 6, 1219 (1989).

[10] J. P. S. Lemos and P. S. Letelier, Class. Quantum Grav. 10, L75 (1993).

[11] J. Bičák, D. Lynden-Bell, and J. Katz, Phys. Rev. D 47, 4334 (1993).

[12] J. Bičák, D. Lynden-Bell, and C. Pichon, Mon. Not. R. Astron. Soc. 265, 126 (1993).

[13] J. P. S. Lemos and P. S. Letelier, Phys. Rev D 49, 5135 (1994).

[14] J. P. S. Lemos and P. S. Letelier, Int. J. Mod. Phys. D 5, 53 (1996).

[15] G. A. González and O. A. Espitia, Phys. Rev. D 68, 104028 (2003).

[16] J. Bičák and T. Ledvinka, Phys. Rev. Lett. 71, 1669 (1993).

[17] G. A. González and P. S. Letelier, Phys. Rev. D 62, 064025 (2000).

[18] D. Vogt and P. S. Letelier, Phys. Rev. D 68, 084010 (2003).

[19] M. Ujevic and P. S. Letelier, Phys. Rev. D 70, 084015 (2004).

[20] G. A. González and P. S. Letelier, Phys. Rev. D 69, 044013 (2004).

[21] D. Vogt and P. S. Letelier, to be published in MNRAS.

[22] M. Miyamoto and R. Nagai, Publ. Astron. Japan 27, 533 (1975)

[23] R. Nagai and M. Miyamoto, Publ. Astron. Japan 28, 1 (1976)

[24] G. Satoh, Publ. Astron. Japan 32, 41 (1980)

[25] T. Ledvinka, J. Bičák, and M. Žofka, in Proceeding of 8th Marcel-Grossmann Meeting in General Relativity, edited by T. Piran (World Scientific, Singapore, 1999)

[26] P. S. Letelier, Phys. Rev. D 60, 104042 (1999).

[27] J. Katz, J. Bičák, and D. Lynden-Bell, Class. Quantum Grav. 16, 4023 (1999).

[28] G. García R. and G. A. González, Phys. Rev. D 69, 124002 (2004).

[29] D. Vogt and P. S. Letelier, Phys. Rev. D 70, 064003 (2004).

[30] G. García-Reyes and G. A. González, Class. Quantum Grav. 21, 4845 (2004).

[31] G. García-Reyes and G. A. González, Phys. Rev. D 70, 104005
(2004).

[32] J. L. Synge, Relativity: The General Theory. (North-Holland, Amsterdam, 1966).

[33] G. Neugebauer and R. Meinel, Phys. Rev. Lett. 75, 3046 (1995).

[34] C. Klein, Class. Quantum Grav. 14, 2267 (1997).

[35] C. Klein and O. Richter, Phys. Rev. Lett. 83, 2884 (1999).

[36] C. Klein, Phys. Rev. D 63, 064033 (2001).

[37] J. Frauendiener and C. Klein, Phys. Rev. D 63, 084025 (2001).

[38] C. Klein, Phys. Rev. D 65, 084029 (2002).

[39] C. Klein, Phys. Rev. D 68, 027501 (2003).

[40] C. Klein, Ann. Phys. (Leipzig) 12, 599 (2003).

[41] V. C. Rubin, J. A. Graham and J. D. P Kenney. Ap. J. 394, L9, (1992).

[42] H. Rix, M. Franx, D. Fisher and G. Illingworth. Ap. J. 400, L5, (1992).

[43] F. Bertola et al. Ap. J. 458, L67 (1996).

[44] F. J. Ernst, Phys. Rev. D 168, 1415 (1968).

[45] C. Klein and O. Richter, Ernst Equations and Riemann Surfaces, Lecture Notes in Physics 685 (Springer) (2005)

[46] A. Papapetrou and A. Hamouni, Ann. Inst. Henri Poincaré 9, 179 (1968)

[47] A. Lichnerowicz, C. R. Acad. Sci. 273, 528 (1971)

[48] A. H. Taub, J. Math. Phys. 21, 1423 (1980)

[49] E. Israel, Nuovo Cimento B 44, 1 (1966)

[50] E. Israel, Nuovo Cimento B 48, 463 (1967)

[51] E. Poisson, A Relativist's Toolkit: The Mathematics of BlackHole Mechanics. (Cambridge University Press, 2004)

[52] S. Chandrasekar, The Mathematical Theory of Black Holes. (Oxford University Press, 1992).

[53] L. D. Landau and E. M. Lifshitz, Fluid Mechanics(AddisonWesley, Reading, MA, 1989).

[54] F. H. Seguin, Astrophys. J. 197, 745 (1975). 